\title{
Autologous TBX-4000-treated Peripheral Blood Mononuclear Cells TBX-3400
}

National Cancer Institute

\section{Source}

National Cancer Institute. Autologous TBX-4000-treated Peripheral Blood Mononuclear

Cells TBX-3400. NCI Thesaurus. Code C154565.

An autologous cell preparation in which the autologous peripheral blood mononuclear cells (PBMCs) are treated ex vivo with TBX-4000, a recombinant TAT-MYC fusion protein, with potential antineoplastic and immunomodulating activities. Upon reintroduction of the TBX-3400 cells into the patient, the generated T-cells may activate the immune system and exert a cytotoxic T-lymphocyte (CTL)-mediated immune response against tumor cells. The TBX-4000 recombinant fusion protein consists of the $\mathrm{N}$-terminal 9 amino acid segment of the HIV TAT protein transduction domain (PTD) fused to the MYC protein. TBX-4000 TAT-MYC rapidly localizes to the nucleus and transiently provides signals to drive proliferation and survival of T-cells. 